\title{
Feature-based Similarity Assessment in Ontology using Fuzzy Set Theory
}

\author{
Parisa D. Hossein Zadeh \\ Dept. of Electrical and Computer Engineering \\ University of Alberta \\ Edmonton, Alberta, Canada \\ dehlehho@ualberta.ca
}

\author{
Marek Z. Reformat \\ Dept. of Electrical and Computer Engineering \\ University of Alberta \\ Edmonton, Alberta, Canada \\ reform@ece.ualberta.ca
}

\begin{abstract}
Semantic Web as an evolution of Web has led to the introduction of new technologies including XML-based formats of representing data on the Web: resource description framework (RDF) and ontology. Similarity assessment of the entities has a fundamental role in processing and analyzing data represented in ontology. In this paper, we propose a technique for determining semantic similarity between pieces of information defined in ontology based on features describing each piece of information. The presented method allows for considering a specific context into the similarity evaluation. The quantitative characterization of similarity at different levels of abstraction in ontology is provided using elements of fuzzy set theory. We show through experiments that the proposed method compares favorably to other measures in terms of human judgment of similarity.
\end{abstract}

Keywords: similarity assessment, semantic similarity, fuzzy set theory, ontology, context-based similarity, RDF triples

\section{INTRODUCTION}

With growing number of web pages and available data on the Web the need has arisen for development of effective tools to manage and facilitate access to information stored on the Web. In this process, one of the challenges is finding the most relevant data to the user's interest. In today's Web, relevancy or in other words similarity is evaluated by keyword matching of the query to the pieces of information on the Web. In Semantic Web [1], as a new paradigm providing a novel vision for data representation on the Web, information is presented within a conceptualization hierarchy referred to as ontology. Ontology is expressed by a formal ontology language as Web Ontology Language (OWL). The language is implemented based on information triples defined in the context of Resource Description Framework (RDF) [2]. Thus, in Semantic Web similarity may be computed using the semantics of concepts in ontology. In fact, evaluating the similarity is a central component of a number of tasks performed in Semantic Web, such as data-mining, reasoning, search engines, information retrieval, clustering, ontology mapping, and ontology translation, see $[3,4]$.

In Semantic Web, every piece of information is presented with RDF triples, which provides a user/machine understandable meaning to every concept. In this paper, the proposed approach determines the semantic similarity in the framework of ontology and RDF data model based on the relationships between concepts. In particular, semantic similarity between concepts is computed by analyzing their interconnections regardless of whether they reside within the same ontology or not. In other words, we aim to determine the similarity by considering all kinds of relations between two concepts including hierarchical $(i s-a)$ and non-hierarchical. Fuzzy set theory [5] is used to quantify the similarity measure at different levels of abstraction in ontology. Furthermore, selection of different types of interconnections according to the defined criteria allows the context to be involved in the similarity assessment.

The rest of the paper is organized as follows: Section II provides an overview of related work in the domain of ontology similarity assessment; Section III discusses background information on ontology and concept's features; Section IV presents the proposed semantic similarity approach and describes how fuzzy set theory is utilized; Section V clarifies the process of similarity assessment in an example and evaluates the performance of our similarity measurement technique in comparison with other relative methods; Section VI discusses the characterization and combination of similarity values obtained at different levels of abstraction in ontology. Finally, the paper is concluded in Section VII.

\section{RELATED WORK}

Below we briefly describe number of approaches on the topic of similarity assessment that are using different models to measure the relatedness of concepts in ontology.

Lexical and syntactical model- this model leverages lexical and syntactical knowledge about the concepts, which is stored in external online repositories such as WordNet [6] as well as description of the text [7-9]. This model focuses on simple word matching without processing and understanding the concepts, thus ignores some important semantics factors.

Structural model- known as path-based methods are based on the structure of taxonomic hierarchy in an ontology such as number of nodes (concepts) separating the two concepts [10, 11]. The method in [10] is based on the formula: 


$$
\text { similarity }=-\log \left(\frac{\text { length }}{2 * D}\right)
$$

where length is the length of the shortest path between two concepts, and $D$ is the maximum depth of the used ontology. In [12], similarity is computed based on the distance of the concepts and their common super concept to the root node:

$$
\text { similarity }=\frac{2 * \operatorname{depth}(l c s)}{\operatorname{depth}\left(e_{1}\right)+\operatorname{depth}\left(e_{2}\right)}
$$

where lcs is the least common super concept (subsume), and depth(e) is the depth of a concept $e$ in the used concept structure.

In general, considering the taxonomy into the similarity measure improves the result to be closer to human similarity judgment. Yet, it still lacks description of the semantic since it only uses the subsumption (is- $a$ ) relations between concepts.

Information-theoretic model- the work that fall under this model calculate relatedness of concepts using the available information about the concepts, which may be found in statistical knowledge and probabilistic model of the domain. In [13] the matching is based on the amount of information needed to describe the commonality of concepts as well as information that describes each concept. In [14], semantic similarity is quantified using shared amount of information between two concepts, which is indicated by the information content of the concepts that subsume them in the taxonomy. The formula used in [14] is:

$$
\text { similarity }=\max _{e \in S\left(e_{1}, e_{2}\right)}[-\log p(e)]
$$

where $p(x)$ is the probability of encountering an instance of concept $x . S\left(e_{1}, e_{2}\right)$ is the set of subsuming concepts of the concepts $e_{1}$ and $e_{2}$. The defined similarity measure in information-theoretic model is usually dependant to an existence of probabilistic information about the concepts in ontology, such as frequency of concepts occurrences in the taxonomy, external corpora, or informativeness of relations between concepts.

Feature-based model- methods based on this model are different from the above approaches by relying on intrinsic relationship between the concepts extracted from ontology. Tversky introduced this model in [15], where a similarity degree is determined based on the features of concepts. The formula used for this purpose is:

$$
\text { similarity }=\frac{|X \cap Y|}{|X \cup Y|+|X-Y|+|Y-X|}
$$

where $\mathrm{X}$ and $\mathrm{Y}$ are sets of features for each of the concepts, and $|$.$| is cardinality of a set.$

A degree of similarity is determined using the modified version of the Tversky's index, known as Dice index [16]:

$$
\text { similarity }=\frac{2 *|X \cap Y|}{|X|+|Y|}
$$

Among all the above models, feature-based model is shown to be an efficient similarity technique to be applied in the framework of ontology and RDF, and it is successfully applied in many applications [17-20]. The underlying reason is that the result from feature-based model is very close to human perception of similarity. Our proposed approach is motivated by feature-based model.

\section{ONTOLOGY, FEATURES AND SIMILARITY}

Ontology consists of a finite set of concepts and the relationships between them in order to describe a certain area of knowledge. Information in ontology is structured in a hierarchical way created by domain experts. This new aspect of knowledge representation is utilized by Semantic Web with the goal of providing an environment that is suitable for machine/user data processing.

In the framework of RDF, information is presented in a triple format of subject, predicate, and object. This data representation introduced by the Semantic Web community leads to an important observation that is a principle idea of the proposed approach: similarity between pieces of information can be determined by analysis of connections between these pieces and other information.

In this paper, we not only consider hierarchical is-a relations but also all types of relations between the concepts. This enables a full range of possible relations, which carries semantics about the concepts under study, to be involved in the similarity measure $[19,21]$. Our approach is also contextaware in which similarity measures can be influenced by a specific context and the features used for describing the contexts. This means determining similarity measures based on a selected subset of connections between two concepts according to the given context.

One of the important aspects of ontology used for Semantic Web applications is that knowledge in ontology is represented at different levels of details starting from an abstract definition level, and finishing at an instance level. The definition level contains general information about concepts, their features and relationships to other concepts. The instance level describes information about individuals or instances of the concepts described in the definition level. In practice, the more abstract concepts are located at higher locations in an ontology. According to the principles in information theory it is known that the more abstract concepts have lower information contents [14]. In other words, interconnections between concepts located in different levels of abstraction can carry different similarity distances between the concepts. It should be emphasized that interconnections contain all types of relations between concepts in ontology.

Semantic distance according to the level of abstraction in ontology can be seen in many similarity assessment approaches, which significantly improves the matching results and make them closer to the human judgment. For example, in information-theoretic models, the information content of the least common super concept plays a key role in measuring the similarity [14]. Also, in structural models of similarity assessment [12] the use of hierarchical information of concepts is necessary for calculating the similarity measure. 


\section{PROPOSED FUZZY SEMANTIC SIMILARITY}

In this paper, similarity of two concepts is determined according to the connections between them and the connections that both concepts share to the same other concepts in ontology. In fact, features of a concept are expressed through the connections of that concept in ontology.

We define ontology as a 3-tuple:

$$
O=\{E, R, f\}
$$

where $E$ is a set of concepts (entities) in ontology $O, R$ is a set of connections between concepts, and $f$ is a function that states if any two concepts are connected or not, i.e., $f\left(e_{1}, e_{2}\right)$.

In real-life scenarios, it is common to assess the similarity between concepts in a specific context. It is demonstrated that context plays an important role in semantic of concepts [22]. In such a case, only a subset of features is taken into the similarity evaluation. In ontology, the context is defined by a single or a set of properties. For example, similarity between a professor and a $\mathrm{PhD}$ Student can be analyzed in the context of their research interests, or published papers. Considering each of these contexts is equivalent to evaluating the relevant defined properties in the ontology, e.g., properties "ResearchInterests" and "Published", respectively.

The semantic similarity between two concepts $A$ and $B$ is defined as follows:

$$
\operatorname{sim}\left(A, B \vdots R_{c}\right)=\frac{n_{c m}\left(A, B \vdots R_{c}\right)}{n\left(A \vdots R_{c}\right)}
$$

$R_{c}$ is a set of connections $\left(R_{c} \subset R\right)$ defined by the context. $n\left(A \vdots R_{c}\right)$ denotes the number of features (connections) of concept $A$ within the context $C . n_{c m}\left(A, B: R_{c}\right)$ is the number of common features between concepts $A$ and $B$ defined within the context $C$. The context $\mathrm{C}$ imposes some constraints on selection of the concept relations.

In a generic scenario of evaluating similarity of two concepts $A$ and $B$ without a specific context, (1) converts into:

$$
\operatorname{sim}(A, B)=\frac{n_{c m}(A, B)}{n(A)}
$$

where the similarity is the ratio of total number of features in common for concepts $A$ and $B$ to the total number of features of concept $A$. It is worth noting that the similarity measure is asymmetric. This is due to the existence of different number of features defining each concept. There exist several empirical evidences with regards to the presence of asymmetric similarity. In [15], it is shown that similarity should not be treated in a symmetric fashion while the direction of asymmetry is dependent on the prominence of the concepts.

Recall that different levels of abstraction in ontology influence the conceptual distances between the concepts. In particular, the farther one travels down in an ontology the conceptual distances decrease. The aforementioned conceptual distance can be observed throughout every ontology level; however, without losing the generality we focus only on the conceptual distances at definition and instance levels of an ontology. For example, the similarity between the items "dolphin" and "shark" (located in the instance level) is intuitively higher than the similarity of their associated super concepts found in the definition level, i.e., "mammal" and "fish", respectively. The similarity defined in (1) uses the level of ontology that the concepts reside in to generate a similarity value.

In order to find a reasonable similarity value we utilize fuzzy set theory based on Zadeh's definition [5]. Let $U$ denote a universal set of similarity values given by (1). Based on two noticeable levels in ontology: "definition" and "instance", we define two types of similarity - a definition level similarity $\operatorname{sim}_{d e f}$, and an instance level similarity $\operatorname{sim}_{\text {ins. }}$. For each of them, we define normalized fuzzy sets low and high for low and high values of similarities, respectively. In general, any number of fuzzy sets can be defined and used. The membership function $\mu_{X}$ for each fuzzy set $X$ is defined in the standard way as:

$$
\mu_{X}: U \rightarrow[0,1]
$$

where $[0,1]$ denotes the interval of real numbers from 0 to 1 inclusive. Once the similarity value is calculated according to (1), then it is evaluated in terms of its degrees of membership in the defined fuzzy sets. Exemplary membership functions for the fuzzy sets low and high are determined based on the results of our preliminary experiments, Fig. 1.a and 1.b. The values for the defined membership functions can be obtained through empirical studies depending on the context of application or in the process of personalization. As stated earlier, concepts at the definition level are more abstract when compared to the concepts at the instance level. Thus, higher similarity value is needed to indicate high similarity.

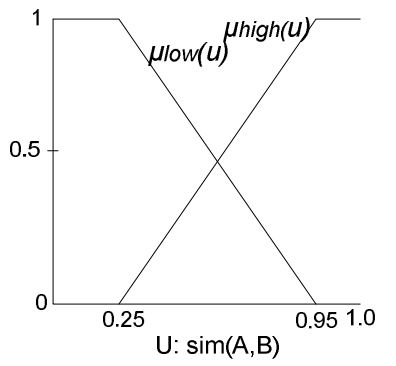

(a)

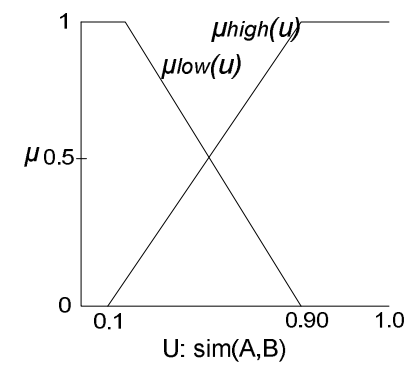

(b)

Figure 1. Membership functions of similarity at the definition level (a), and the instance level (b).

\footnotetext{
${ }^{1}$ In this example, "dolphin" and "shark" are assumed to be actual instances in the ontology, and should not be confused to be concepts containing other sub concepts.
} 
On the contrary, the similarity value required for implying similarity of concepts at the instance level is more relaxed. For example, at the definition level any similarity value below 0.25 is "labeled" as low, while any measure above 0.25 is high to some degree. At the instance level, any value above 0.10 is "labeled" as high to a degree.

For a given pair of concepts $(A, B)$, we find their similarity $\operatorname{sim}\left(A, B: R_{C}\right)$ using (1). According to the level of ontology (i.e., definition or instance) that the concepts $(A, B)$ are associated with, membership degrees of $\operatorname{sim}(A, B)$ to fuzzy sets low and high are obtained, which are indicated by $\operatorname{sim}_{\text {def }}\left(A, B: R_{c}\right)$ and $\operatorname{sim}_{\text {ins }}\left(A, B: R_{c}\right)$. It worth mentioning that level of belongingness for each concept can be determined from the syntax of ontology language and the annotations used in the ontology. For example, using Fig. 1.a and 1.b and the similarity value 0.3 , calculated using (1), the degrees of membership of the similarity in the low and high fuzzy sets are shown in Table I.

Table I. Membership degrees of $\operatorname{sim}(A, B)$

\begin{tabular}{|c|c|c|c|}
\hline \multirow{2}{*}{$\operatorname{sim}\left(A, B: R_{c}\right)$} & Levels & $\mu_{\text {low }}(u)$ & $\mu_{\text {high }}(u)$ \\
\hline \multirow{2}{*}{0.3} & $\operatorname{sim}_{\text {def }}\left(A, B: R_{c}\right)$ & 0.93 & 0.07 \\
\cline { 2 - 4 } & $\operatorname{sim}_{\text {ins }}\left(A, B: R_{c}\right)$ & 0.75 & 0.25 \\
\hline
\end{tabular}

There are number of advantages of applying fuzzy approach in the process of similarity assessment. Firstly, it is simple and intuitive. Secondly, it gives a more human linguistic description of similarity judgment. Thirdly and most importantly, the values for low and high fuzzy sets, or any number of fuzzy sets, are easily customized to the needs of the user.

\section{EXPERIMENTS AND RESULTS}

The presented ontology in this paper is an integration and modification of three existing ontologies: ontology of concepts from academic research ${ }^{2}$, ontology of university concepts ${ }^{3}$, and computing science concepts. In the developed ontology, two concepts of professor and PhDStudent are defined as well as their properties, including connections to other concepts at definition level and their links to instances at the instance level of ontology. A snapshot the definition level of the ontology is shown in Fig. 2. a and Fig. 2. b.

Fig. 3 depicts a small fragment of the ontology including each particular concept with its associated properties. The concept professor has three connections (relations) to the other concept Publication while PhDStudent has two properties associated with Publication. At the instance level, it can be seen that there exist an instance of each concept professor and PhD Student: professor X and PhDStudentY. Professor X has 21 instances of published journal papers, and PhDStudent $Y$ has published 18 journal papers in total from which 8 of them are shared with the concept professorX. In Fig. 3, the titles of the journal papers are indicated by "P\#".

\footnotetext{
${ }^{2}$ ka.owl: http://protegewiki.stanford.edu/wiki/Protege_Ontology_Library\# OWL_ontologies

${ }^{3}$ univ-bench.owl: http://swat.cse.lehigh.edu/projects/lubm/

${ }^{4}$ http://www.owl-ontologies.com/ComputingOntology.owl
}

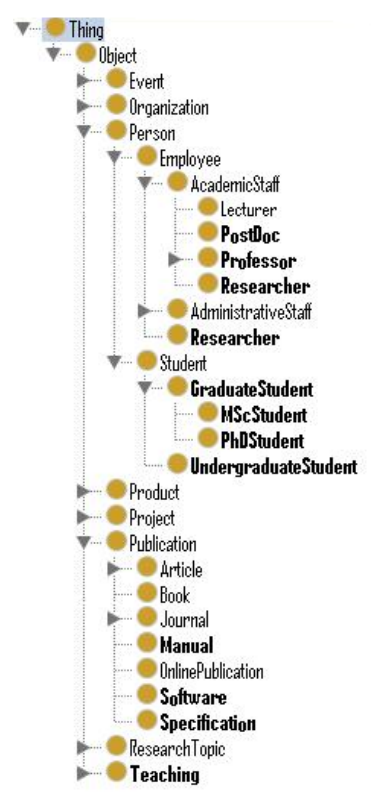

(a)

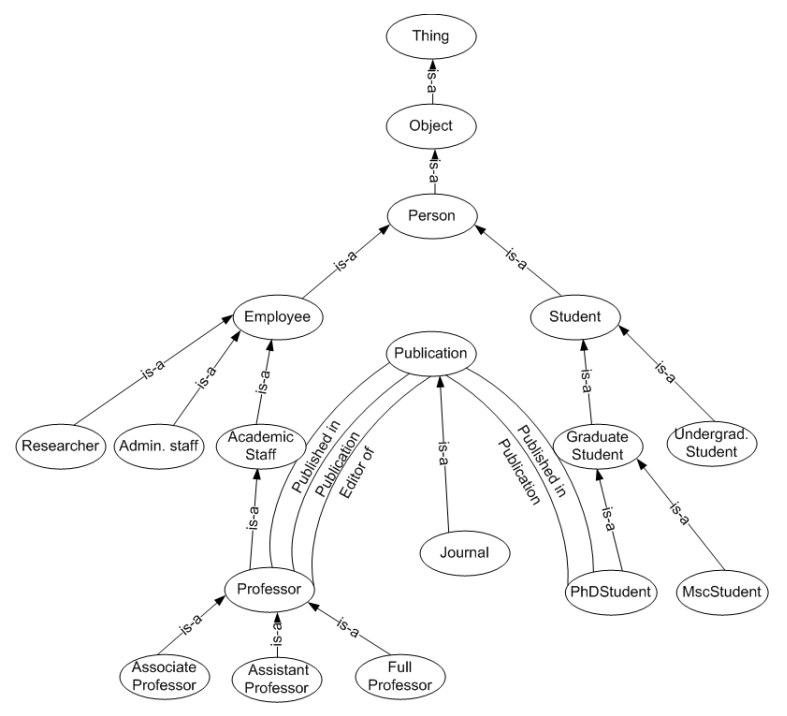

(b)

Figure 2. A snapshot (a) and a fragment (b) of the developed ontology.

Let us calculate the similarity of the pair of concepts professor and PhDStudent at the definition level as well as the instance level according to the proposed approach. Based on the number of features for each concept as shown in Fig. 3, and assuming that the set of defined properties in the context of publication is \{Published in, Publication, Editor of\}, the similarity of the pair (Professor, PhDStudent) at the definition level in the context of publication is calculated in the following way:

$$
\begin{aligned}
& \operatorname{sim}_{\text {def }}(\text { professor }, \text { PhDStudent }: \text { Publication }) \\
& =\frac{n_{c m}(\text { professor }, \text { PhDStudent }: \text { Publication })}{n(\text { professor }: \text { Publication })}=\frac{2}{3}=0.66
\end{aligned}
$$




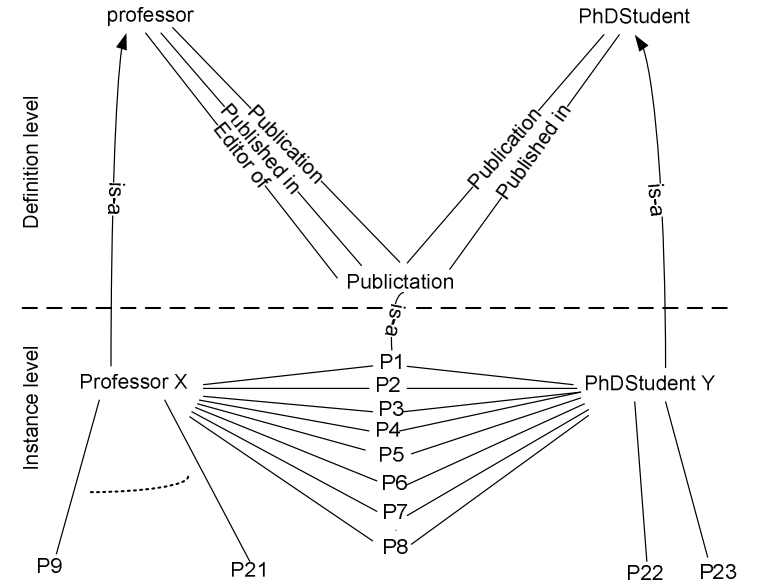

Figure 3. Concepts professor and PhDStudent in definition level and their instances ProfessorX and PhDStudentY.

This can be explained in the following way: the concept professor has three properties defined in the context of publication, including Publication, Published in, and Editor of (see Fig. 3). It can also be seen that the concept professor shares two properties (Publication and Published in) within this context with the concept PhDStudent.

Likewise, the similarity of the pair (professorX, PhDStudentY) at the instance level within the context of publication is determined as:

$\operatorname{sim}_{\text {ins }}$ (professorX, PhDStudentY:Publication)

$=\frac{n_{c m}(\text { professor } X, P h D S t u d e n t Y \vdots \text { Publication })}{n(\text { professor } X \vdots \text { Publication })}$

$=\frac{8}{21}=0.38$

It should be noted that the obtained values describe the similarity of a professor to a PhDStudent. Similarity of a PhDStudent to a professor would give a different value. This indicates the asymmetric feature of the approach, and is reflected by the prominence of the concept. Such a result is in the agreement with Tversky's claim [15].

In the next step, since the concepts belong to the definition and instance levels of ontology, degrees of membership of the obtained similarities are computed based on membership functions presented in Fig. 1.a and 1.b, respectively. The results are shown in Table II.

Table II. Degrees of membership of similarity for professor and PhDStudent

\begin{tabular}{|c|c|c|c|}
\hline $\operatorname{sim}\left(\boldsymbol{A}, \boldsymbol{B}: \boldsymbol{R}_{c}\right)$ & similarity value & $\mu_{\text {low }}(u)$ & $\mu_{\text {high }}(u)$ \\
\hline $\operatorname{sim}_{\text {def }}\left(A, B: R_{c}\right)$ & 0.66 & 0.42 & 0.58 \\
\hline $\operatorname{sim}_{\text {ins }}\left(A, B: R_{c}\right)$ & 0.38 & 0.65 & 0.35 \\
\hline
\end{tabular}

As can be seen in Table II, the computed similarity value of professor and PhDStudent at the definition level is equal to 0.66 , while similarity of their instances - at the instance level - is 0.38 . As it can be observed, degrees of membership to the low and high fuzzy sets of both similarities are different. If we perform a simple defuzzification in the form of an $\alpha$-cut for $\alpha=0.5$ for both similarities, we obtain that similarity at the definition level is high, while at the instance level is low. However, if $\alpha=0.65$ then similarity is low at the instance level, while similarity at the definition level is undetermined.

In order to evaluate our approach, we selected one existing method related to each similarity model as described in Section II. In order to make the comparison fair and meaningful the similarity value of our method is the contextfree value obtained in (2), and without applying the fuzzy memberships. In other words, the shown result of our method is the raw calculated similarity value. The ontology built for this paper, presented in Fig. 2, is used in the comparison. The evaluation of results is performed using human judgment of similarity for which we averaged the similarities given by 25 graduate students for each pair of concepts, see Table III.

As it can be observed, the proposed similarity assessment performs quite well comparing with other techniques as well as human judgment. An interesting observation can be made here: the result from our proposed method is closest to the ones from Tversky and Dice index, which are also featurebased similarity models. Although the results from featurebased similarity model give a reasonably close value to the human judgment but still there is a room to be further improved. We believe that this improvement can be accomplished by taking the abstraction level of concepts in ontology into the consideration, which is performed in our approach by utilizing fuzzy set theory.

Table III. Comparison of multiple similarity assessment methods

\begin{tabular}{|c|c|c|c|c|c|c|c|c|}
\hline sim. model & $\begin{array}{c}\text { Edit } \\
\text { distance }\end{array}$ & $\begin{array}{c}\text { Shortest } \\
\text { Path } \\
\text { length }\end{array}$ & $\begin{array}{c}\text { Leacock \& } \\
\text { Chodorow } \\
{[\mathbf{1 0 ]}}\end{array}$ & $\begin{array}{c}\text { Wu \& } \\
\text { Palmer } \\
{[\mathbf{1 2}]}\end{array}$ & $\begin{array}{c}\text { Tversky } \\
\text { index } \\
{[\mathbf{1 5}]}\end{array}$ & $\begin{array}{c}\text { Dice } \\
\text { index } \\
{[\mathbf{1 6}]}\end{array}$ & $\begin{array}{c}\text { Our } \\
\text { method }\end{array}$ & $\begin{array}{c}\text { Human } \\
\text { judgment }\end{array}$ \\
\hline $\begin{array}{c}\text { professor, } \\
\text { phd_student }\end{array}$ & 0.09 & 0.17 & 0.48 & 0.25 & 0.33 & 0.56 & 0.61 & 0.60 \\
\hline $\begin{array}{c}\text { professor, } \\
\text { researcher }\end{array}$ & 0.11 & 0.50 & 0.95 & 0.75 & 0.88 & 0.90 & 0.90 & 0.72 \\
\hline $\begin{array}{c}\text { researcher, } \\
\text { phd_student }\end{array}$ & 0.09 & 0.20 & 0.48 & 0.25 & 0.40 & 0.67 & 0.71 & 0.82 \\
\hline $\begin{array}{c}\text { professor, } \\
\text { admin_staff }\end{array}$ & 0.05 & 0.33 & 0.78 & 0.57 & 0.11 & 0.30 & 0.47 & 0.34 \\
\hline
\end{tabular}




\section{DISCUSSION}

The determination of similarity at two different levels definition and instance - creates an opportunity to mimic human's way of similarity assessment of two items in which a person uses his/her knowledge about the categories that these two items belong to. It seems quite plausible to assume that once the items are classified, in other words categorized, the first similarity assessment is done at the level of abstraction of concepts. Next, the person looks at the details of the items under evaluation and adjusts his/her first assessment. At the same time it is reasonable to state that the first assessment has some influence on the second assessment. In an attempt to model such a process we propose the following procedure for applying the influence of similarity obtained at each level of abstraction.

As stated, the similarity obtained at the definition level$\operatorname{sim}_{d e f}-$ is fuzzified. Two common membership functions are used here: $\mu_{\text {low }}$ and $\mu_{\text {high }}$. The result, i.e., two membership values $\mu_{\text {low }}\left(\operatorname{sim}_{d e f}\right)$ and $\mu_{\text {high }}\left(\operatorname{sim}_{d e f}\right)$ are combined with the instance level similarity - simins. One of the possible ways of calculating this is presented here. The final similarity value can be obtained as:

$$
\text { similarity }=\left\{\begin{array}{l}
\max \left\{0, \operatorname{sim}_{\text {ins }}-\alpha * \mu_{\text {low }}\left(\operatorname{sim}_{\text {def }}\right)\right\} \\
\text { when } \mu_{\text {low }}\left(\operatorname{sim}_{\text {def }}\right) \geq \mu_{\text {high }}\left(\operatorname{sim}_{\text {def }}\right) \\
\max \left\{1, \operatorname{sim}_{\text {ins }}+\beta^{*} \mu_{\text {high }}\left(\operatorname{sim}_{\text {def }}\right)\right\} \\
\text { when } \mu_{\text {low }}\left(\operatorname{sim}_{\text {def }}\right)<\mu_{\text {high }}\left(\operatorname{sim}_{\text {def }}\right)
\end{array}\right.
$$

where $\alpha$ and $\beta$ represent levels of influence of $\operatorname{sim}_{d e f}$ on the final similarity value. If the membership degree of $\operatorname{sim}_{\text {def }}$ in low fuzzy set is larger or equal to its membership degree in high fuzzy set, then the $\operatorname{sim}_{\text {ins }}$ is decreased by the user-defined fraction $(\alpha)$ of $\operatorname{sim}_{\text {def }}$. In the other case, $\operatorname{sim}_{\text {ins }}$ is increased by the user-defined fraction $(\beta)$ of $\operatorname{sim}_{d e f}$. The final calculated similarity in (3) can be further translated into a human-friendly linguistic description.

Let us take the results form the example presented in the previous section. The obtained values are: 0.42 for $\mu_{\text {low }}\left(\operatorname{sim}_{\text {def }}\right)$ and 0.58 for $\mu_{\text {high }}\left(\operatorname{sim}_{\text {def }}\right)$. Therefore, the second option in (3) is applicable. For the value of $\beta=0.5$ - moderate influence of similarity at the definition level - we obtain the value of similarity equal to 0.64 . For $\beta=0.75-$ higher influence of definition-based similarity - the similarity value is 0.79 . For $\beta=1.0$ the similarity is a summation of both similarities at the definition and instance levels (of course, if the sum exceeds 1.0 the similarity value assumes 1.0 ).

\section{CONCLUSION}

In this paper, a novel approach for analyzing the semantic similarity of concepts in ontology is proposed. A feature-based similarity assessment method is presented while it incorporates semantic into the measure by evaluating features of concepts in ontology. The proposed approach allows for determining similarity when specific contexts are taken into consideration. Fuzzy set theory in defining membership degrees of the obtained similarity values according to the level of abstraction of concepts in ontology is adopted. Realizing the effect of abstraction level of a concept in similarity measure, different fuzzy sets are defined to distinguish membership degrees associated with each abstraction level.

\section{REFERENCES}

[1] T. B. Lee, J. Hendler and O. Lassila, "The semantic web," Sci. Am., vol. 284, pp. 34-43, 2001.

[2] O. Lassila and R. Swick. Resource description framework (RDF) model and syntax specification. W3C Tech. Reports and Publications [http://www.w3.org/TR/1999/REC-rdf-syntax-19990222]. 1999.

[3] W. Wei, P. Barnaghi and A. Bargiela, "Rational Research model for ranking semantic entities," Inf. Sci., 2011.

[4] J. R. G. Pulido, S. B. F. Flores, R. C. M. Ramirez and R. A. Diaz, "Eliciting ontology components from semantic specific-domain maps: Towards the next generation web," in Web Congress, 2009. LEWEB'09. Latin American, 2009, pp. 224-229.

[5] L. A. Zadeh, "Similarity relations and fuzzy orderings," Inf. Sci., vol. 3, pp. 177-200, April, 1971.

[6] G. A. Miller, R. Beckwith, C. Fellbaum, D. Gross and K. J. Miller, "Introduction to wordnet: An on-line lexical database*," International Journal of Lexicography, vol. 3, pp. 235-244, 1990.

[7] F. Giunchiglia, M. Yatskevich and P. Shvaiko, "Semantic matching: Algorithms and implementation," in 2007, pp. 1-38.

[8] P. A. V. Hall and G. R. Dowling, "Approximate String Matching," ACM Comput.Surv., vol. 12, pp. 381-402, December, 1980.

[9] G. Hirst and D. St-Onge, "Lexical chains as representations of context for the detection and correction of malapropisms," WordNet: An Electronic Lexical Database, vol. 13, pp. 305-332, 1998.

[10] C. Leacock and M. Chodorow, "Combining local context with WordNet similarity for word sense identification," in WordNet: A Lexical Reference System and its Application, MIT Press, 1998, pp. 265-283.

[11] R. Rada, H. Mili and E. Bicknell, "Development and application of a metricon semantic nets," IEEE Transaction on Systems, Man and Cybernets, vol. 19, pp. 17-30, 1989.

[12] Z. Wu and M. Palmer, "Verbs semantics and lexical selection," in Proceedings of the 32nd Annual Meeting on Association for Computational Linguistics, Las Cruces, New Mexico, 1994, pp. 133138.

[13] D. Lin, "An information-theoretic definition of similarity," in Proceedings of the Fifteenth International Conference on Machine Learning, 1998, pp. 296-304.

[14] P. Resnik, "Using information content to evaluate semantic similarity in a taxonomy," in IJCAI, 1995, pp. 448-453.

[15] A. Tversky, "Features of similarity," Psychol. Rev., vol. 84, pp. 327-352, $7,1977$.

[16] W. B. Frakes and R. Baeza-Yates, "Information Retrieval: Data Structures and Algorithms, PTR Prentice-Hall," Inc. , Eaglewood Cliffs, New Jersey, vol. 7632, 1992.

[17] H. Tang, H. Maitre and N. Boujemaa, "Similarity measures for satellite images with heterogeneous contents," in Urban Remote Sensing Joint Event, 2007, 2007, pp. 1-9.

[18] H. Eidenberger, "Evaluation and analysis of similarity measures for content-based visual information retrieval," Multimedia Systems, vol. 12, pp. 71-87, 2006.

[19] P. Giuseppe, "A semantic similarity metric combining features and intrinsic information content," Data Knowl. Eng., vol. 68, pp. 1289 1308, November, 2009. 
[20] C. Pesquita, D. Faria, A. O. Falclao, P. Lord and F. M. Couto, "Semantic similarity in biomedical ontologies," PLoS Computational Biology, vol. 5, pp. e1000443, 2009.

[21] L. Mazuel and N. Sabouret, "Semantic relatedness measure using object properties in an ontology," The Semantic Web-ISWC 2008, pp. 681-694, 2008.

[22] S. Patwardhan and T. Pedersen, "Using WordNet Based Context Vectors to Estimate the Semantic Relatedness of Concepts," pp. 1-8, apr, 2006. 